\title{
¿Son los pigmentos fotosintéticos buenos indicadores de la relación del nitrógeno, fósforo y potasio en frijol ejotero?
}

\section{Are photosynthetic pigments good indicators of the nitrogen-phosphorus- potassium nutritional state in beans?}

\author{
Esteban Sánchez ${ }^{1 *}$, Juan Manuel Ruiz², Luis Romero², Pablo Preciado-Rangel ${ }^{3}$; María Antonia \\ Flores-Córdova ${ }^{4}$, César Márquez-Quiroz ${ }^{5}$ \\ ${ }^{1}$ Centro de Investigación en Alimentación y Desarrollo, A.C. Unidad Delicias. Av. Cuarta Sur 3820, Fracc. Vencedores del \\ Desierto, CP. 33089, Cd. Delicias, Chihuahua. México. \\ ${ }^{2}$ Departamento de Fisiología Vegetal, Facultad de Ciencias, Universidad de Granada. Granada, España. \\ ${ }^{3}$ Instituto Tecnológico de Torreón. Torreón, Coahuila, México. \\ ${ }^{4}$ Facultad de Ciencias Agrotecnológicas, Universidad Autónoma de Chihuahua. Chihuahua, México. \\ ${ }^{5}$ Universidad Juárez Autónoma de Tabasco. Villahermosa, Tabasco. México. \\ ${ }^{*}$ Autor de correspondencia: esteban@ciad.mx
}

Artículo científico recibido: 20 de febrero de 2018 aceptado: 13 de junio de 2018

RESUMEN. El objetivo fue conocer si los pigmentos foliares son buenos indicadores del estado nutricional del $\mathrm{N}, \mathrm{P}$ y $\mathrm{K}$ en plantas de frijol ejotero. Se realizaron tres estudios independientes, en el primero, el $\mathrm{N}$ se aplicó a la solución nutritiva en la forma de $\mathrm{NH}_{4} \mathrm{NO}_{3}$ en dosis crecientes de 0 a $24.0 \mathrm{mM}$ de $\mathrm{N}$. En el segundo experimento, el $\mathrm{P}$ se aplicó en la forma $\mathrm{H}_{3} \mathrm{PO}_{4}$ en dosis crecientes de 0 a $8.0 \mathrm{mM}$ de $\mathrm{P}$, y en el tercer experimento, el $\mathrm{K}$ se aplicó en la forma $\mathrm{KSO}_{4}$ en dosis crecientes de 0 a $16.0 \mathrm{mM}$ de $\mathrm{K}$. Los parámetros analizados en tejido foliar fueron: concentración de $\mathrm{N}, \mathrm{P}$ y K , biomasa, concentración de pigmentos foliares y sus relaciones. Los resultados indican que la aplicación de $\mathrm{N}$ favoreció la acumulación de clorofila a, b y carotenos, presentándose la máxima concentración en la dosis de 18 $\mathrm{mM}$, de la misma manera la aplicación de $\mathrm{P}$ favoreció la acumulación de pigmentos, presentando la dosis de $4.0 \mathrm{mM}$, la mayor acumulación; mientras que la aplicación de $\mathrm{K}$ presentó un comportamiento inverso al $\mathrm{N}$ y $\mathrm{P}$, presentando la mayor acumulación de pigmentos en la dosis de $1.0 \mathrm{mM}$. La concentración de clorofila a y b, y clorofila total tuvo correlación positiva y significativa con el estado nutricional del N. Los modelos matemáticos polinomial pudieran ser un instrumento para la toma de decisiones para el manejo nutricional sustentable de la fertilización de N, P y K.

Palabras clave: Phaseolus vulgaris L., manejo nutricional, fertilización, deficiencia, frijol ejotero, toxicidad

ABSTRACT. The objective was to determine whether leaf pigments are good indicators of the nutrient status of $\mathrm{N}, \mathrm{P}$ and $\mathrm{K}$ in bean plants. Three independent studies were conducted; in the first, $\mathrm{N}$ was applied to the nutrient solution in the form of $\mathrm{NH}_{4} \mathrm{NO}_{3}$ in increasing doses from 0 to $24.0 \mathrm{mM} \mathrm{N}$. In the second experiment, $\mathrm{P}$ was applied in $\mathrm{H}_{3} \mathrm{PO}_{4}$ form in increasing doses from 0 to $8.0 \mathrm{mM} \mathrm{P}$, and in the third experiment, $\mathrm{K}$ was applied in $\mathrm{KSO}_{4}$ form in increasing doses from 0 to $16.0 \mathrm{mM} \mathrm{K}$. The parameters analyzed in leaf tissue were: concentration of $\mathrm{N}, \mathrm{P}$ and $\mathrm{K}$, biomass, concentration of foliar pigments and their ratios. The results indicate that $\mathrm{N}$ application favored the accumulation of chlorophyll $\mathrm{a}, \mathrm{b}$ and carotenes, presenting the maximum concentration at the $18 \mathrm{mM}$ dose; in the same way, the $\mathrm{P}$ application favored the accumulation of pigments, with the $4.0 \mathrm{mM}$ dose having the greatest accumulation, while the $\mathrm{K}$ application exhibited an inverse behavior to $\mathrm{N}$ and $\mathrm{P}$, having the highest accumulation of pigments with the $1.0 \mathrm{mM}$ dose. The concentration of chlorophyll $\mathrm{a}, \mathrm{b}$ and total had a positive and significant correlation with $\mathrm{N}$ nutrient status. The polynomial mathematical models could be an instrument for decision making for sustainable nutrient management of $\mathrm{N}, \mathrm{P}$ and $\mathrm{K}$ fertilization.

Key words: Phaseolus vulgaris L., nutrient management, fertilization, deficiency, beans, toxicity 


\section{INTRODUCCIÓN}

La fotosíntesis es el principal proceso fisiológico por el que las plantas responden a los cambios en las condiciones ambientales (Calatayud y Barreno et al. 2004). Los pigmentos fotosintéticos son importantes para las plantas principalmente para la cosecha de luz y producción de poder reductor como ATP y NADPH (Masoudi-Sadaghiani et al. 2011). Sin embargo, diferencias en la actividad de pigmentos fotosintéticos, pueden ser considerados un síntoma de una fertilización inadecuada, o un desbalance nutricional, no obstante, otros factores ambientales como la temperatura, salinidad o sequía pueden disminuir la actividad de los pigmentos fotosintéticos (Tanaka et al. 2008).

Hoy en día, es común la aplicación de la fertilización nitrogenada, fosfórica y potásica en la actividad hortícola mundial (Ling et al. 2017). El nitrógeno $(N)$, fósforo $(P)$ y potasio $(K)$ son los mayores factores limitantes del crecimiento y rendimiento de los cultivos (Parry et al. 2005). Los nutrientes minerales tienen funciones esenciales y específicas en el metabolismo de las plantas, como activadores de reacciones enzimáticas, osmorreguladores y constituyentes de estructuras orgánicas (Latsague et al. 2014). Actualmente el $\mathrm{N}$ es el nutriente más usado y demandado para el crecimiento de las plantas cultivadas, además de un componente esencial de proteínas y ácidos nucleicos, cuando la cantidad de $\mathrm{N}$ en el medio de cultivo, no se encuentra disponible en un rango óptimo, el crecimiento se reduce (Kraiser et al. 2011).

La capacidad fotosintética de las hojas está relacionada con el contenido de $\mathrm{N}$, principalmente porque las proteínas del ciclo de Calvin y tilacoides representan la mayoría del nitrógeno foliar (Latsague et al. 2014). Un adecuado suplemento de $\mathrm{N}$ es esencial en el establecimiento de la máxima capacidad de demanda y la capacidad fotosintética; pero un uso excesivo de $\mathrm{N}$ provoca excesiva expansión del área foliar, que se correlaciona de forma inversa con la capacidad fotosintética. Recientes investigaciones indican una estrecha relación entre la concentración de clorofila foliar y el contenido de N, lo que tiene sentido, debido a que la mayoría del $\mathrm{N}$ foliar está en las moléculas de clorofila (Torres-Netto et al. 2005). Dado que los pigmentos fotosintéticos tienen el potencial de detectar deficiencias de $\mathrm{N}$, también es prometedor como herramienta para mejorar el manejo de la fertilización nitrogenada (Balasu-bramanian et al. 2000).

El fósforo es el segundo elemento nutritivo, después del $\mathrm{N}$, que limita el crecimiento de las plantas y la productividad de los cultivos, este nutriente forma parte de muchas moléculas que participan en procesos básicos de las plantas (Sánchez et al. 2009). El P en su forma inorgánica $(\mathrm{Pi})$ está involucrado en el control de muchas reacciones enzimáticas, que regulan procesos metabólicos (Salinas et al. 2013). Los efectos de la deficiencia de $\mathrm{P}$ incluyen: la reducción en el número de hojas y la pérdida de la eficiencia fotosintética. También participa en procesos metabólicos esenciales para el crecimiento normal, como la fotosíntesis (Salinas et al. 2012); debido a que influye en la estabilidad de la clorofila (Bojovic y Stojanovic 2005).

El $\mathrm{K}$ es un nutriente esencial para las plantas. A pesar de su naturaleza catiónica, su comportamiento es similar a la que presentan el $\mathrm{P}$ y $\mathrm{N}$, redistribuyéndose con facilidad de los órganos maduros a los juveniles por su movilidad y baja afinidad con los ligandos orgánicos de los que fácilmente se intercambia (Latsague et al. 2014). En general, la deficiencia de N, P y K afecta la fotosíntesis y el crecimiento (Muñoz-Huerta et al. 2013). Pero hay poca información del efecto del N, $\mathrm{P}$ y $\mathrm{K}$ en dosis elevadas sobre la concentración de pigmentos fotosintéticos. Por lo que el objetivo del presente trabajo fue estudiar la respuesta de dosis deficientes y elevadas de N, P y K sobre la concentración de diferentes pigmentos fotosintéticos en hojas de frijol ejotero cv. Strike.

\section{MATERIALES Y MÉTODOS}

\section{Manejo del cultivo y diseño experimental}

Las semillas del frijol (Phaseolus vulgaris L.) cv. Strike se germinó en una cámara de crecimiento 
a $28{ }^{\circ} \mathrm{C}$ por $48 \mathrm{~h}$. Posteriormente, se trasladaron a una cámara de cultivo en la Universidad de Granada, España, bajo condiciones ambientales con humedad relativa de 60 a $80 \%$, temperatura $28 / 22$ ${ }^{\circ} \mathrm{C}$ (día/noche), fotoperiodo de $16 / 8$ h (día/noche) e intensidad luminosa de $350 \mu \mathrm{mol} \mathrm{m} \mathrm{m}^{-2} \mathrm{~s}^{-1}$. Las plantas se pusieron en macetas individuales de 25 $\mathrm{cm}$ de diámetro superior y $25 \mathrm{~cm}$ de altura de $8 \mathrm{~L}$, llenadas con vermiculita.

Se realizaron tres experimentos diferentes, en el primero, el nitrógeno se aplicó a la solución nutritiva en forma de $\mathrm{NH}_{4} \mathrm{NO}_{3}$ en dosis de $\mathrm{N} 1=1.5$ $\mathrm{mM}, \mathrm{N} 2=3.0 \mathrm{mM}, \mathrm{N} 3=6.0 \mathrm{mM}, \mathrm{N} 4=12.0 \mathrm{mM}$, $\mathrm{N} 5=18.0 \mathrm{mM}$ y $\mathrm{N} 6=24.0 \mathrm{mM}$ de $\mathrm{N}$. En el segundo experimento, el fósforo se aplicó en la forma de $\mathrm{H}_{3} \mathrm{PO}_{4}$ en dosis de $\mathrm{P} 1=0.5 \mathrm{mM}, \mathrm{P} 2=1.0 \mathrm{mM}$, $\mathrm{P} 3=2.0 \mathrm{mM}, \mathrm{P} 4=4.0 \mathrm{mM}, \mathrm{P} 5=6.0 \mathrm{mM}$ y $\mathrm{P} 6=$ $8.0 \mathrm{mM}$ de $\mathrm{P}$ y en el tercer experimento, el potasio se aplicó en la forma $\mathrm{KSO}_{4}$ en dosis de $\mathrm{K} 1=1.0$ $\mathrm{mM}, \mathrm{K} 2=2.0 \mathrm{mM}, \mathrm{K} 3=4.0 \mathrm{mM}, \mathrm{K} 4=8.0 \mathrm{mM}$, $\mathrm{K} 5=12.0 \mathrm{mM}$ y $\mathrm{K} 6=16.0 \mathrm{mM}$ de $\mathrm{K}$.

El ciclo de cultivo tuvo una duración de $60 \mathrm{~d}$. Los primeros $30 \mathrm{~d}$ a partir del trasplante y antes de la aplicación de los tratamientos experimentales, las plantas recibieron una solución nutritiva completa de Hoagland ( $\mathrm{pH}$ 6.0-6.1), la cual estuvo compuesta de $6 \mathrm{mM}$ de $\mathrm{NH}_{4} \mathrm{NO}_{3}, 1.6 \mathrm{mM}$ de $\mathrm{K}_{2} \mathrm{HPO}_{4}$, $2.4 \mathrm{mM}$ de $\mathrm{K}_{2} \mathrm{SO}_{4}, 4.0 \mathrm{mM}$ de $\mathrm{CaCl}_{2} \cdot \mathrm{p}_{2} \mathrm{H}_{2} \mathrm{O}, 1.4$ $\mathrm{mM}$ de $\mathrm{MgSO}_{4}, 5 \mu \mathrm{M}$ de Fe-EDDHA, $2 \mu \mathrm{M}$ de $\mathrm{MnSO}_{4} \cdot \mathrm{H}_{2} \mathrm{O}, 1.0 \mu \mathrm{M}$ de $\mathrm{ZnSO}_{4} \cdot 7 \mathrm{H} 2 \mathrm{O}, 0.25 \mu \mathrm{M}$ de $\mathrm{CuSO}_{4} \cdot 5 \mathrm{H}_{2} \mathrm{O}, 0.3 \mu \mathrm{M}$ de $\left(\mathrm{NH}_{4}\right) 6 \mathrm{Mo}_{7} \mathrm{O}_{24} \cdot 4 \mathrm{H}_{2} \mathrm{O}$ y $0.5 \mu \mathrm{M}$ de $\mathrm{H}_{3} \mathrm{BO}_{3}$ preparada con agua destilada, el pH de la solución osciló entre 5.5 y 6.0 (Sánchez et al. 2004). Durante los últimos $30 \mathrm{~d}$ del ciclo de cultivo, se aplicaron en la solución nutritiva los tratamientos de N, P y K, respectivamente. El diseño experimental fue un diseño completamente al azar con seis repeticiones por tratamiento y cuatro plantas por repetición.

\section{Muestreo vegetal}

A los $60 \mathrm{~d}$ después de germinadas ( $\mathrm{ddg}$ ) se muestrearon las plantas completas, las cuales se encontraban en la fase fenológica de desarrollo completo y madurez del fruto. El material fresco se utilizó para la cuantificación de biomasa, pigmentos foliares: clorofila a, clorofila b y carotenos, mientras que el material seco se utilizó para la determinación de N, P y K. Para cada variable analizada se utilizaron cuatro repeticiones por tratamiento.

\section{Análisis Vegetal}

La producción de biomasa foliar se obtuvo del promedio de peso por planta con base en materia seca (g). Mientras que la concentración foliar de N, $\mathrm{P}$ y $\mathrm{K}$ en $\%$ se obtuvo de las hojas, las cuales se trituraron hasta obtener un polvo para determinar el contenido total de $\mathrm{N}$ con un analizador orgánico elemental (FLASH 2000 analizador, Thermo Scientific ${ }^{T M}$ ). El contenido total de $\mathrm{P}$ se determinó con un espectrofotómetro UV-VIS por el método del molibdatovanadato, después de digerir las muestras con $\mathrm{HNO}_{3}$ concentrado (65\%) y $\mathrm{H}_{2} \mathrm{O}_{2}$ (30\%); en tanto que el contenido total de $\mathrm{K}$ se determinó con el método de espectroscopía de absorción atómica (ASS, Thermo Scientific ${ }^{T M}$ ) (Sadzawka et al. 2007).

Los pigmentos fotosintéticos se determinaron con el método descrito por Wellburn (1994), para lo cual se pesó entre 0.2 y $0.3 \mathrm{~g}$ de hojas frescas en taleolas, con diámetro de $7 \mathrm{~mm}$. A las que se le añadieron $10 \mathrm{ml}$ de metanol puro $(\mathrm{CH} 3 \mathrm{OH})$ e incubó a temperatura ambiente en oscuridad por $24 \mathrm{~h}$. Para luego medir la absorbancia a $470 \mathrm{~nm}$ (carotenoides), $653 \mathrm{~nm}$ (clorofila b, chl b) y $666 \mathrm{~nm}$ (clorofila a, chl a). El cálculo de la concentración de pigmentos se realizó con las siguientes fórrmulas de Wellburn (1994):

Chl a: $\left[15.65 \cdot\left(\mathrm{A}_{666}\right)-7.34 \cdot\left(\mathrm{A}_{653}\right)\right]$

$$
\frac{C h l a \cdot V_{1} \cdot p_{1}}{\left(p_{2} \cdot 2^{2} \cdot n\right)}
$$

Chl b: $\left[27.05 \cdot\left(\mathrm{A}_{653}\right)-11.21 \cdot\left(\mathrm{A}_{666}\right)\right]$

$$
\frac{C h l b \cdot V_{1} \cdot p_{1}}{\left(p_{2} \cdot 2^{2} \cdot n\right)}
$$

Carotenoides: [1000.( $\left.\mathrm{A}_{470}\right)-2.86 \cdot(\mathrm{Chl}$ a) -129.2 (Chl b)] / 221

$$
\frac{\text { Carotenoides } \cdot V_{1} \cdot p_{1}}{\left(p_{2} \cdot 2^{2} \cdot n\right)}
$$


Donde: V1: Volumen de la extracción, p1: peso en $\mathrm{g}$ por taleola, p2: peso total en $\mathrm{g}, \mathrm{n}$ : número de taleolas y $r^{2}$ : diámetro de la taleola. Las concentraciones de clorofila $a$, clorofila $b$ y carotenoides se expresaron como $\mu \mathrm{g} \mathrm{cm}^{-2}$.

\section{Análisis estadístico}

Los datos obtenidos se sometieron a análisis de varianza. Para determinar diferencias entre medias de los tratamientos se utilizó la prueba LSD al $95 \%$. Los niveles de significancia de ambos análisis se expresaron como: ${ }^{*} p<0.05,{ }^{*} p<<0.01,{ }^{* * *} p$ $<0.001$ y NS (no significativo).

\section{RESULTADOS}

En la Tabla 1, se presenta la producción de biomasa foliar en función de las dosis de $\mathrm{N}$ aplicado, sobresaliendo el tratamiento N3 con la máxima biomasa foliar en relación con el tratamiento N6 que presentó la mínima acumulación de biomasa. Destaca el tratamiento N6 con la mayor concentración de $\mathrm{N}$ total mientras que el tratamiento N1 tuvo la menor concentración. En la Figura 1 , se observa que los distintos tratamientos de $\mathrm{N}$ aumentaron el contenido de la clorofila $\mathrm{a}, \mathrm{b}$ y total, así como los carotenos, presentando los máximos valores el tratamiento $\mathrm{N} 5$, con incrementos superiores al $80 \%$ con respecto a las mínimas concentraciones del tratamiento N1.

En la Tabla 2, se observa efecto del aumento de la dosis de $\mathrm{N}$ y la concentración de pigmentos fotosintéticos, siendo mayores las concentraciones de clorofila a y b, en relación con los carotenos. La aplicación de diferentes dosis de nitrógeno influyó en el contenido de clorofila a, b y carotenos, así como, en la relación de los pigmentos, con disminución de los contenidos de clorofilas y carotenos con la dosis N6. En la Tabla 3, se presenta la correlación entre el estado nutricional del $\mathrm{N}$, pigmentos foliares y biomasa, destacando la concentración de clorofila a y b, y clorofila total con correlación positiva y significativa con el estado nutricional del $\mathrm{N}$. Con respecto al fósforo (Tabla 1) se observa que el tratamiento P3 tuvo la máxima biomasa foliar en relación al tratamiento
P1 que tuvo la mínima acumulación de biomasa, también se observa que el tratamiento P6 tuvo la mayor concentación de $\mathrm{P}$ total.

Se encontraron diferencias significativas en la concentración de pigmentos fotosintéticos por efecto de las diferentes dosis de $\mathrm{P}$, sobresaliendo la dosis P4 con la máxima concentración de clorofila a, b y carotenos, con incrementos del 35\%, 33\% y $37 \%$ con relación a la mínima concentración de la dosis P1 (Figura 1). Las diferentes dosis de P tuvieron un comportamiento parecido al $\mathrm{N}$, ya que a medida que se incrementó la dosis de $\mathrm{P}$, se tuvo acumulación de clorofila a y b, y carotenos, siendo el tratamiento $\mathrm{P} 4$ el que presentó el mayor contenido de pigmentos fotosintéticos.

En la Tabla 2, se presenta el efecto de la aplicación de las dosis de $\mathrm{P}$ sobre los pigmentos fotosintéticos. La relación clorofila a/clorofila b, varió de 2.86 a 3.26 , la relación clorofila a/carotenos fue de 6.06 a 7.05, la relación clorofila b/carotenos fue de 1.94 a 2.19 y la relación clorofila total/carotenos varió de 8.01 a 9.21. Así mismo, presentó cocientes más bajos en las relaciones de pigmentos por efecto de la aplicación de dosis de $\mathrm{P}$ en comparación con las dosis de N. Las dosis deficientes de P (P1 y P2) presentaron un comportamiento similar a las dosis elevadas de P (P5 y P6), siendo la dosis considerada como óptima desde el punto de vista de pigmentos fotosintéticos la $\mathrm{P} 4$, ya que presentó la máxima acumulación de clorofila a y b, y carotenos. En la Tabla 3 , se presenta la matriz de correlación entre el estado nutricional del $\mathrm{P}$, pigmentos foliares y biomasa, destacando que no hay correlación positiva y significativa entre los diferentes pigmentos foliares y el estado nutricional del P.

Con respecto al Potasio, en la Tabla 1 se presenta la producción de biomasa foliar en función de los tratamientos de $\mathrm{K}$, sobresaliendo la dosis $\mathrm{K} 5$ con la máxima biomasa foliar en relación a la dosis $\mathrm{K} 1$ que presentó la mínima acumulación de biomasa. En la misma Tabla, se presenta el efecto de las dosis de $\mathrm{K}$ sobre la acumulación de $\mathrm{K}$ total foliar, destacando la dosis $\mathrm{K} 6$ con la mayor concentración de $\mathrm{K}$ total en comparación con la menor concentración obtenida en la dosis K1. Por otro lado, el K pre- 
Tabla 1. Efecto de los tratamientos de N, P y K sobre el estado nutricional del N, P y K, y la acumulación de biomasa foliar.

\begin{tabular}{|c|c|c|c|}
\hline \multirow[t]{2}{*}{ Tratamientos } & \multicolumn{3}{|c|}{ Nitrógeno } \\
\hline & $\mathrm{N}$ total $(\%)$ & Biomasa foliar (g planta $^{-1}$ & p.s.) \\
\hline N1 & 2.75 & 0.90 & \\
\hline $\mathrm{N} 2$ & 3.08 & 1.24 & \\
\hline N3 & 3.98 & 1.42 & \\
\hline N4 & 4.35 & 1.26 & \\
\hline N5 & 4.61 & 0.90 & \\
\hline N6 & 4.75 & 0.80 & \\
\hline \multirow[t]{3}{*}{ Significancia } & $* *$ & $*$ & \\
\hline & \multicolumn{3}{|c|}{ Fósforo } \\
\hline & $\mathrm{P}$ total $(\%)$ & Biomasa foliar (g planta $^{-1}$ & p.s.) \\
\hline P1 & 0.62 & 1.10 & \\
\hline P2 & 0.68 & 1.22 & \\
\hline P3 & 0.83 & 1.42 & \\
\hline P4 & 0.90 & 1.36 & \\
\hline P5 & 0.99 & 1.28 & \\
\hline P6 & 1.14 & 1.20 & \\
\hline \multirow[t]{3}{*}{ Significancia } & $* *$ & $*$ & \\
\hline & \multicolumn{3}{|c|}{ Potasio } \\
\hline & $\mathrm{K}$ total $(\%)$ & Biomasa foliar (g planta $^{-1}$ & p.s.) \\
\hline K1 & 1.63 & 1.21 & \\
\hline $\mathrm{K} 2$ & 1.68 & 1.38 & \\
\hline K3 & 1.99 & 1.39 & \\
\hline K4 & 2.35 & 1.42 & \\
\hline K5 & 3.78 & 1.51 & \\
\hline K6 & 4.65 & 1.30 & \\
\hline Significancia & ** & * & \\
\hline
\end{tabular}

sentó un comportamiento inverso al $\mathrm{N}$ y $\mathrm{P}$ (Figura 1 ), siendo las dosis deficientes las que favorecieron la mayor acumulación de pigmentos fotosintéticos en relación con las dosis elevadas de K. Observándose que la dosis deficiente $\mathrm{K} 1$ tuvo la máxima concentración de clorofila a y b, y carotenos, con incrementos de 57,49 y $48 \%$ con relación a la dosis $\mathrm{K} 4$ que tuvo la mínima concentración de pigmentos. Para la aplicación de las diferenciadas dosis de $\mathrm{K}$ sobre la relación de los pigmentos fotosintéticos se observó que los contenidos de pigmentos fotosintéticos se redujeron a partir de la dosis K5 (Tabla 2).

La correlación entre el estado nutricional del $\mathrm{K}$, pigmentos foliares y biomasa, indica correlación positiva y significativa entre los diferentes pigmentos foliares y el estado nutricional del K (Tabla 3). En la Tabla 4, se presentan las ecuaciones del modelo polinomial y sus coeficientes de determinación $\left(R^{2}\right)$. Para el $N$, los modelos de regresión cuadrática (polinomial) fueron significativos para clorofila a, b, y carotenos, presentando el valor máximo de $\mathrm{R}^{2}$ la concentración de carotenos. Para el $\mathrm{P}$, la clorofila a tuvo la $\mathrm{R}^{2}$ más alta, mientras que para el $\mathrm{K}$ fue la clorofila $\mathrm{b}$ que tuvo los mayores valores. Los modelos de regresión polinomial determinados representan el comportamiento de los pigmentos fotosintéticos estudiados en base a las dosis de N, P y K aplicados.

\section{DISCUSIÓN}

En trabajos previos, se ha observado que la acumulación de pigmentos fotosintéticos en las plantas, es un indicador de producción de biomasa y del estado nutricional de N (Lamrani et al. 1996). El tratamiento N3 presentó la máxima producción de biomasa foliar, con incremento del $43 \%$ en relación con el tratamiento $\mathrm{N} 6$ que tuvo el valor mínimo (Tabla 1). La aplicación de $6 \mathrm{mM}$ de $\mathrm{N}$ (N3) es el mejor tratamiento para incrementar la producción de biomasa foliar en plantas de frijol ejotero. Por lo que los tratamientos por debajo de 
Tabla 2. Efecto de los tratamientos de $\mathrm{N}, \mathrm{P}$ y K sobre la relación entre pigmentos fotosintéticos en frijol ejotero. Los datos son medias $(n=6)$.

\begin{tabular}{|c|c|c|c|c|c|}
\hline Tratamiento de $\mathrm{N}$ & Chla/Chlb & Total Chla+Chlb & Chla/Caro & Chlb/Caro & Total/Caro \\
\hline $\mathrm{N} 1$ & 1.95 & 258.93 & 7.70 & 3.94 & 11.64 \\
\hline $\mathrm{N} 2$ & 2.26 & 414.62 & 9.60 & 4.23 & 13.84 \\
\hline N3 & 2.68 & 532.28 & 8.86 & 3.29 & 12.16 \\
\hline N4 & 2.05 & 1101.36 & 10.88 & 5.29 & 16.17 \\
\hline N5 & 2.77 & 2092.56 & 7.84 & 2.82 & 10.66 \\
\hline N6 & 1.78 & 1155.22 & 15.69 & 8.79 & 24.49 \\
\hline Significancia & $*$ & $* * *$ & $* *$ & $*$ & $* *$ \\
\hline Tratamiento de $\mathrm{P}$ & Chla/Chlb & Total Chla+Chlb & Chla/Caro & Chlb/Caro & Total/Caro \\
\hline P1 & 2.86 & 453.85 & 6.30 & 2.19 & 8.50 \\
\hline P2 & 3.12 & 516.25 & 6.06 & 1.94 & 8.01 \\
\hline P3 & 3.26 & 601.99 & 7.05 & 2.16 & 9.21 \\
\hline P4 & 2.95 & 695.94 & 6.12 & 2.07 & 8.19 \\
\hline P5 & 3.29 & 555.80 & 6.59 & 1.99 & 8.58 \\
\hline P6 & 2.95 & 463.73 & 6.41 & 2.16 & 8.57 \\
\hline Significancia & $*$ & $* * *$ & $* *$ & $* *$ & $* *$ \\
\hline Tratamiento de $\mathrm{K}$ & Chla/Chlb & Total Chla+Chlb & Chla/Caro & Chlb/Caro & Total/Caro \\
\hline $\mathrm{K} 1$ & 2.98 & 2142.48 & 7.47 & 2.50 & 9.97 \\
\hline $\mathrm{K} 2$ & 2.34 & 1261.63 & 7.51 & 3.20 & 10.72 \\
\hline K3 & 2.38 & 1218.98 & 7.68 & 3.22 & 10.91 \\
\hline K4 & 2.54 & 971.95 & 6.32 & 2.48 & 8.81 \\
\hline K5 & 2.46 & 1116.02 & 6.09 & 2.47 & 8.56 \\
\hline K6 & 2.87 & 1299.12 & 4.98 & 1.73 & 6.72 \\
\hline Significancia & $*$ & $* * *$ & $* *$ & $* *$ & $* *$ \\
\hline $\begin{array}{l}\text { Clorofila a (Chla), } \\
\text { (Chla/Chlb), Clorc } \\
\text { (Chla/Caro), Relac } \\
\text { (Total/Caro). * a }\end{array}$ & fila & b), Carc & 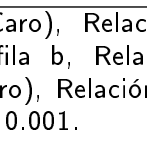 & Cloro & $\begin{array}{l}\text { Clorofila b } \\
\text { /Carotenos } \\
\text { /Carotenos }\end{array}$ \\
\hline
\end{tabular}

N3 (N1 y N2) pueden ser considerados como deficientes de $\mathrm{N}$, mientras que las dosis por encima de N3 son de elevadas a tóxicas por la disminución en la producción de biomasa foliar. Los pigmentos fotosintéticos son importantes para la captura de energía solar durante la fotosíntesis, por lo que diferencias en la actividad clorofílica son síntomas de una fertilización inapropiada de $\mathrm{N}$ ó un desbalance nutricional, aunque factores ambientales, como temperatura, salinidad o sequía, pueden disminuir la actividad clorofílica (Latsague et al. 2014). La clorofila foliar se relaciona con la concentración de $\mathrm{N}$ foliar (Zhang et al. 2013), por lo que puede ser un buen indicador de la concentración de $\mathrm{N}$ foliar (Naeem et al. 2009).

De acuerdo a la mayoría de las investigaciones, la relación entre clorofila a y b es de $3: 1$, los cuales varían en función del crecimiento y desarrollo de la planta, del cultivar y de factores ambientales (Bojovic y Stojanovic 2005). El contenido de carotenos fue bajo en comparación con los valores de clorofila, mientras que el contenido de carotenos mostró poca variación en función de la nutrición mineral (Tabla 1). Estudios previos demuestran que el aumento del $\mathrm{N}$ en la rizósfera aumento las concentraciones foliares de clorofila a y $b$, así como la clorofila total, independientemente de la dosis de $\mathrm{P}$ aplicada. Las concentraciones totales de clorofila se correlacionaron con el nivel de fertilización de P. EI caroteno y los licopenos reflejaron la influencia de las dosis crecientes de $\mathrm{N}$, mientras que $\mathrm{P}$ no afectó a estos pigmentos.

El fósforo es un macronutriente esencial, que está involucrado en diversos procesos fisiológicos y bioquímicos de las plantas, siendo parte integral del metabolismo de la energía, componente de ácidos nucleicos y de las membranas (Hawkesford et al. 1995, Salinas et al. 2012). La baja disponibilidad de $P$ es uno de los principales factores que limitan la producción de cultivos en la mayor parte del planeta (Raghothama y Karthikeyan 2005). Niveles excesivos de $\mathrm{P}$ disponible en el medio pueden 
Tabla 3. Matriz de correlación entre el estado nutricional del $N, P$ y K, pigmentos foliares y biomasa.

\begin{tabular}{|c|c|c|c|c|c|c|}
\hline & Ntotal & Chla & Chlb & Chltotal & Caro & Biomasa \\
\hline Ntotal & 1.0 & $\begin{array}{l}0.76^{*} \\
p=0.05\end{array}$ & $\begin{array}{l}0.87^{*} \\
\mathrm{p}=0.02\end{array}$ & $\begin{array}{l}0.80^{*} \\
\mathrm{p}=0.05\end{array}$ & $\begin{array}{l}0.57 \\
p=0.22\end{array}$ & $\begin{array}{l}-0.17 \\
p=0.73\end{array}$ \\
\hline Chla & & 1.0 & $\begin{array}{l}0.95 * * \\
p=0.0036\end{array}$ & $\begin{array}{l}0.99 * * \\
p<0.0001\end{array}$ & $\begin{array}{l}0.95^{* *} \\
p=0.0028\end{array}$ & $\begin{array}{l}-0.36 \\
p=0.47\end{array}$ \\
\hline Chlb & & & 1.0 & $\begin{array}{l}0.97^{* *} \\
\mathrm{p}=0.0010\end{array}$ & $\begin{array}{l}0.82^{*} \\
\mathrm{p}=0.0452\end{array}$ & $\begin{array}{l}-0.45 \\
p=0.36\end{array}$ \\
\hline Chltotal & & & & 1.0 & $\begin{array}{l}0.92 \\
p=0.0075\end{array}$ & $\begin{array}{l}-0.39 \\
p=0.43\end{array}$ \\
\hline Caro & & & & & 1.0 & $\begin{array}{l}-0.28 \\
p=0.58\end{array}$ \\
\hline Biomasa & & & & & & 1.0 \\
\hline & Ptotal & Chla & Chlb & Chltotal & Caro & Biomasa \\
\hline Ptotal & 1.0 & $\begin{array}{l}0.12 \\
p=0.81\end{array}$ & $\begin{array}{l}0.08 \\
p=0.88\end{array}$ & $\begin{array}{l}0.11 \\
p=0.83\end{array}$ & $\begin{array}{l}0.05 \\
p=0.92\end{array}$ & $\begin{array}{l}0.27 \\
p=0.60\end{array}$ \\
\hline Chla & & 1.0 & $\begin{array}{l}0.94 * * \\
p=0.0051\end{array}$ & $\begin{array}{l}0.99 * * \\
\mathrm{p}<0.0001\end{array}$ & $\begin{array}{l}0.94^{* *} \\
\mathrm{p}=0.0050\end{array}$ & $\begin{array}{l}0.88^{* *} \\
p=0.0206\end{array}$ \\
\hline Chlb & & & 1.0 & $\begin{array}{l}0.96^{* *} \\
\mathrm{p}=0.0017\end{array}$ & $\begin{array}{l}0.96^{*} \\
p=0.0019\end{array}$ & $\begin{array}{l}0.72 \\
p=0.1057\end{array}$ \\
\hline Chltotal & & & & 1.0 & $\begin{array}{l}0.95^{* *} \\
\mathrm{p}=0.0027\end{array}$ & $\begin{array}{l}0.85^{*} \\
\mathrm{p}=0.03\end{array}$ \\
\hline Caro & & & & & 1.0 & $\begin{array}{l}0.69 \\
p=0.12\end{array}$ \\
\hline Bıomasa & Ktotal & Chla & Chlb & Chltotal & Caro & $\begin{array}{l}1.0 \\
\text { Biomasa }\end{array}$ \\
\hline Ktotal & 1.0 & $\begin{array}{l}-0.30 \\
p=0.55\end{array}$ & $\begin{array}{l}-0.47 \\
p=0.34\end{array}$ & $\begin{array}{l}-0.34 \\
p=0.50\end{array}$ & $\begin{array}{l}0.23 \\
p=0.65\end{array}$ & $\begin{array}{l}0.21 \\
p=0.67\end{array}$ \\
\hline Chla & & 1.0 & $\begin{array}{l}0.96 * * \\
\mathrm{p}=0.0019\end{array}$ & $\begin{array}{l}0.99 * * \\
\mathrm{p}<0.0001\end{array}$ & $\begin{array}{l}0.83^{*} \\
\mathrm{p}=0.0385\end{array}$ & $\begin{array}{l}v-0.85^{*} \\
p=0.0313\end{array}$ \\
\hline Chlb & & & 1.0 & $\begin{array}{l}0.97 * * \\
\mathrm{p}=0.0007\end{array}$ & $\begin{array}{l}0.68 \\
p=0.13\end{array}$ & $\begin{array}{l}-0.77 \\
p=0.06\end{array}$ \\
\hline Chltotal & & & & 1.0 & $\begin{array}{l}0.80^{*} \\
\mathrm{p}=0.0519\end{array}$ & $\begin{array}{l}-0.84 \\
p=0.0362\end{array}$ \\
\hline Caro & & & & & 1.0 & $\begin{array}{l}-0.81 \\
p=0.0462\end{array}$ \\
\hline Biomasa & & & & & & 1.0 \\
\hline
\end{tabular}

interferir con la absorción de Zn o Fe y en consecuencia, pueden alterar la producción de biomasa (Hawkesford et al. 1995). Mientras que el fósforo es un importante elemento nutricional que puede limitar el crecimiento de las plantas (Raghothama 1999). Al respecto se encontró que los tratamientos de $\mathrm{P}$ afectaron la producción de biomasa foliar (Tabla 1), destacando el tratamiento P3 $(2.0 \mathrm{mM}$ de $\mathrm{P}$ ) con incremento del $15 \%$ en relación con el tratamiento $\mathrm{P} 6$ que presentó la menor biomasa foliar. Los tratamientos por debajo de P3 (P1 y P2) pueden ser considerados deficientes de $\mathrm{P}$; mientras que las dosis por encima de $\mathrm{P} 3$ pudieran considerarse elevadas a tóxicas por la disminución de biomasa fo- liar.

El fósforo es el principal sustrato de la fotosíntesis y tiene funciones estructurales en las membranas (Vance et al. 2003), también regula el metabolismo energético de las plantas debido a su presencia en el ATP, ADP, AMP y en moléculas pirofosfato (Xu et al. 2007). Deficiencias de $\mathrm{P}$ conducen a cambios en procesos morfológicos, fisiológicos y bioquímicos como decremento de la relación brote/raíz (Rubio y Lynch 2007). Al respecto, Yaryura et al. (2009) menciona que la deficiencia de $\mathrm{P}$ produjo disminución de los valores de reflexión entre 500 y $650 \mathrm{~nm}$, con mayores contenidos de antocianina, clorofila y daño al fotosistema II. 
Sánchez et al.
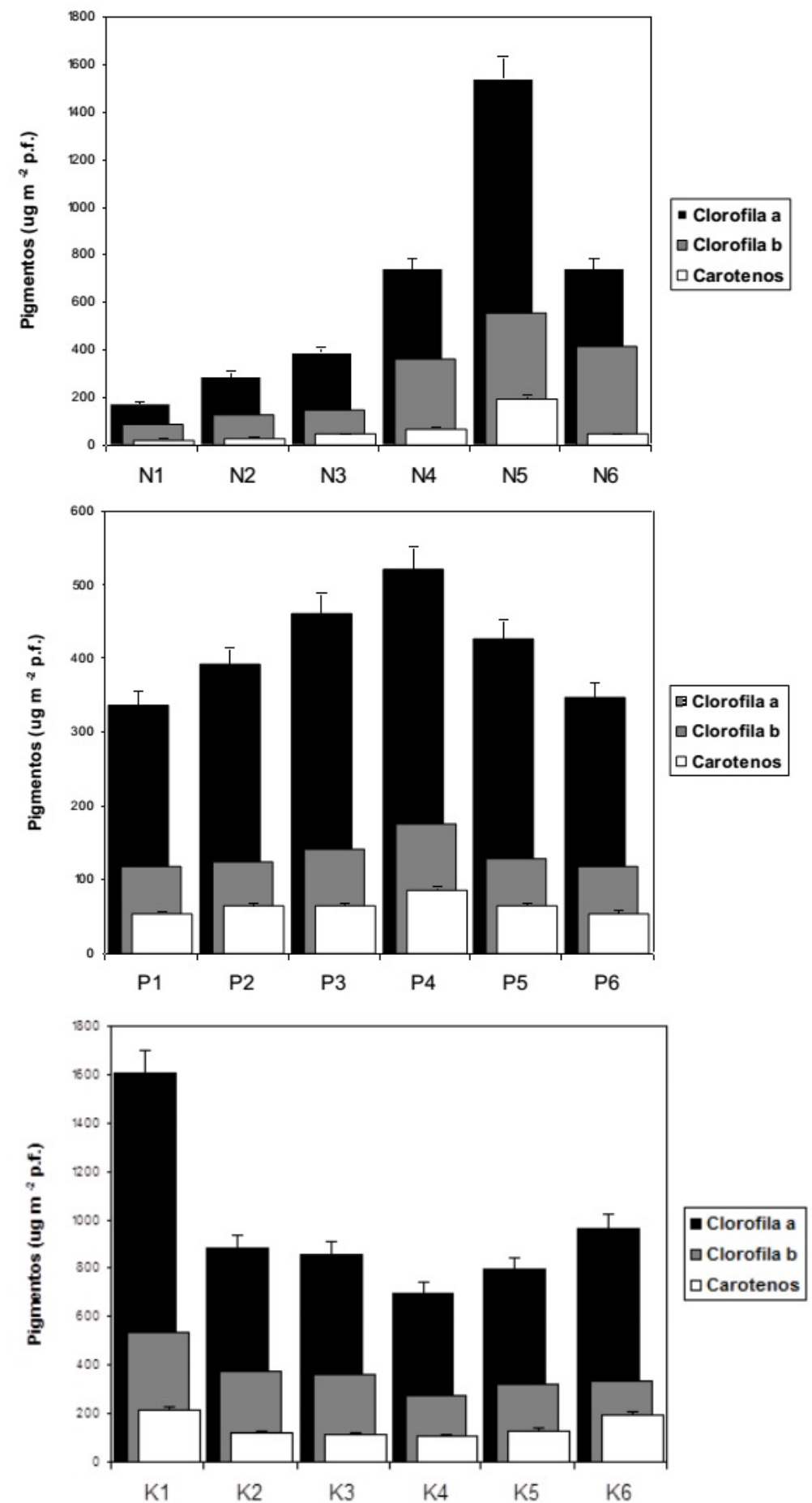

Figura 1. Efecto de los tratamientos de $\mathrm{N}, \mathrm{P}$ y $\mathrm{K}$ sobre la concentración de pigmentos fotosintéticos en frijol ejotero. Los datos son medias \pm error estándar $(n=6)$ 
Tabla 4. Ecuaciones del modelo polinomial y coeficientes de determinación $\left(R^{2}\right)$ para pigmentos fotosintéticos en respuesta a la aplicación de N, P y K en frijol ejotero.

\begin{tabular}{ccc}
\hline & Nitrógeno & \\
\hline Pigmento & Modelo de regresión cuadrática (polinomial) & $\mathrm{R}^{2}$ \\
Clorofila a & $\mathrm{y}=-31.866 \times 2+421.59 \times-347.91$ & 0.5898 \\
Clorofila b & $\mathrm{y}=-5.8293 \times 2+59.308 \times-51.28$ & 0.3460 \\
Carotenos & $\mathrm{y}=-3.33 \times 2+112.91 \times-63.194$ & 0.7874 \\
\hline \multicolumn{3}{c}{ Fósforo } \\
\hline Pigmento & Modelo de regresión cuadrática (polinomial) & $\mathrm{R}^{2}$ \\
Clorofila a & $\mathrm{y}=-23.668 \times 2+171.84 \times+171.07$ & 0.8755 \\
Clorofila b & $\mathrm{y}=-3.4455 \times 2+24.797 \times+29.941$ & 0.6930 \\
Carotenos & $\mathrm{y}=-6.2579 \times 2+45.128 x+71.344$ & 0.6025 \\
\hline \multicolumn{3}{c}{ Potasio } \\
\hline Pigmento & Modelo de regresión cuadrática (polinomial) & $\mathrm{R}^{2}$ \\
Clorofila a & $\mathrm{y}=88.135 \times 2-720.84 x+2153.4$ & 0.9083 \\
Clorofila b & $\mathrm{y}=16.146 \times 2-115.03 \times+304.06$ & 0.9371 \\
Carotenos & $\mathrm{y}=20.198 \times 2-177.5 x+682.81$ & 0.9271 \\
\hline
\end{tabular}

Al igual que el $\mathrm{N}$ y $\mathrm{P}$, el $\mathrm{K}$ es el nutriente que se necesita para un adecuado crecimiento de las plantas. Se encontró que los tratamientos de $\mathrm{K}$ afectaron la producción de biomasa foliar (Tabla 1), siendo el tratamiento $\mathrm{K} 3$ el que presentó el mayor valor, con incrementos de 36 y $15 \%$, en comparación con los valores mínimos de los tratamientos $\mathrm{K} 1$ y $\mathrm{K} 6$, respectivamente. La aplicación de $4.0 \mathrm{mM}$ de $\mathrm{K}$ (K3) y de $8.0 \mathrm{mM}(\mathrm{K} 4)$ fueron los tratamientos con mayor producción de biomasa foliar en plantas de frijol ejotero, mientras que los tratamientos por debajo de los tratamientos $\mathrm{K} 1$ y $\mathrm{K} 2$ son deficientes de $\mathrm{K}$. mientras que las dosis de $\mathrm{K} 5$ y $\mathrm{K} 6$, se consideran tóxicas por la disminución del crecimiento de las plantas de frijol. Estos resultados coinciden con los publicados por Salinas et al. (2012) y Latsague et al. (2014) quienes indican que el $\mathrm{N}$ y $\mathrm{P}$ tienen influencia sobre el contenido de clorofila. Mientras que Daughtry (2000) indican que el $\mathrm{N}$ es un elemento estructural de la clorofila y de moléculas de proteína, y por lo tanto afecta la formación de cloroplastos y la acumulación de clorofila. La influencia del $\mathrm{P}$ sobre la formación de pigmentos verdes en las hojas depende de la concentración, debido a que afecta la estabilidad de la clorofila en las plantas. Aunque el $\mathrm{N}$ es el elemento mineral más importante en el proceso de biosíntesis de la clorofila, una dosis elevada reduce la vida de las hojas, incrementa su sensibilidad, y baja su resistencia a enfermedades, lo que conduce a un decremento del contenido de clorofila foliar. Mien- tras que la deficiencia de $\mathrm{P}$ inhibe el crecimiento vegetal y la síntesis de clorofila. Por lo que el contenido de clorofila puede utilizarse como indicador de estrés (Tejada-Zarco 2004). Estudios previos, indican efecto de la fertilización con N, P y K sobre los parámetros bioquímicos de las hojas de $B$. corallina, principalmente en el contenido de clorofila a y b (Latsague et al. 2014). Todos los elementos macro-metabólicos tienen influencia en el desarrollo de plantas en general y su superficie foliar es influenciada por el nitrógeno. Al respecto, Bojovic y Stojanovic (2005) reportan que el contenido de las distintas dosis de N, P y K indican diferencias en las concentraciones de pigmentos fotosintéticos, con mayores contenidos con las dosis con mayores contenidos de $\mathrm{N}$ y $\mathrm{P}$, mientras que para el $\mathrm{K}$, fueron mayores en las dosis bajas.

La deficiencia de N, P O K suprimió el crecimiento, aceleró la senescencia y disminuyó las concentraciones de los pigmentos cloroplásticos (Kumar Tewari et al. 2007). La deficiencia de los principales componentes estructurales, como el nitrógeno $(N)$ o el fósforo $(P)$, se espera que reduzca la frecuencia de los portadores de electrones de los sistemas de transporte de electrones (Grossman y Takahashi 2001). La deficiencia de potasio (K) retarda la absorción de CO2 debido al cierre estomático, lo que conduce a la acumulación del poder reductor (NADPH), sobre-reducción de los portadores del transporte de electrones y la generación del 
radical superóxido (O2-) y otras especies de oxígeno reactivo (Grossman y Takahashi 2001). La senescencia, es una consecuencia importante de la deficiencia de $\mathrm{N} \circ \mathrm{P}$ y el estrés oxidativo comparten síntomas comunes, como la pérdida de pigmentos y proteínas cloroplásticas, la peroxidación lipídica y las alteraciones de la membrana, lo que conduce a una disminución progresiva de la capacidad fotosintética (Kumar Tewari et al. 2007). La aplicación de $\mathrm{N}$ favoreció la acumulación de clorofila $\mathrm{a}, \mathrm{b}$ y carotenos, presentando la máxima concentración la dosis de $18 \mathrm{mM}$, de la misma manera la aplicación de $\mathrm{P}$ favoreció la acumulación de pigmentos, presentando la dosis de $4.0 \mathrm{mM}$, la mayor acumulación; mientras que para el $\mathrm{K}$ presentó un comportamiento inverso al $\mathrm{N}$ y $\mathrm{P}$, presentando la mayor acumulación de pigmentos la dosis de $1.0 \mathrm{mM}$.

La clorofila es el pigmento más importante en las hojas, y responsable de su verdor. El contenido de clorofila puede utilizarse como indicador del estado nutricional del nitrógeno, ya que éste es un elemento esencial en la síntesis de proteínas fotosintéticas (Demotes-Mainard et al. 2008). Al respecto, la concentración de clorofila a y b, y total tuvieron una correlación positiva y significativa con el estado nutricional del N (Tabla 3). Por lo que, los pigmentos fotosintéticos son buenos indicadores del estado nutricional del $\mathrm{N}$, pero no del estado nutricional del $\mathrm{P}$ y K . El modelo matemático polinomial fue el que mejor explicó las curvas de pigmentos fo- tosintéticos, debido a que tuvo los meyores valores del coeficiente de determinación (Tabla 4). La aplicación de la dosis $\mathrm{N} 6$, puede considerarse una dosis tóxica de $\mathrm{N}$, que favorecio la acumulación de $\mathrm{H}_{2} \mathrm{O}_{2}$ en tejido foliar (Sánchez et al. 2000) y generó una necrosis foliar que afecto los pigmentos fotosintéticos y el crecimiento de las plantas de frijol ejotero. Al igual que el $\mathrm{N}$, las dosis elevadas de $\mathrm{P}$ y $\mathrm{K}$, fueron tóxicas, debido a que disminuyeron el crecimiento de la parte aérea, probablemente debido al estrés oxidativo que generó condiciones de toxicidad de $\mathrm{P}$ y $\mathrm{K}$, lo que puede verse reflejado en la disminución del contenido de pigmentos foliares.

\section{CONCLUSIONES}

La aplicación de $\mathrm{N}$ favoreció la acumulación de clorofila a, b y carotenos, presentando la máxima concentración la dosis de $18 \mathrm{mM}$, de la misma manera la aplicación de $\mathrm{P}$ favoreció la acumulación de pigmentos, presentando la dosis de $4.0 \mathrm{mM}$, la mayor acumulación; mientras que para el K presentó un comportamiento inverso al $\mathrm{N}$ y $\mathrm{P}$, presentando la mayor acumulación de pigmentos la dosis de 1.0 mM. La concentración de clorofila a y b, y clorofila total tuvieron una correlación positiva y significativa con el estado nutricional del N. Finalmente, se concluye que el modelo matemático polinomial puede ser un instrumento para la toma de decisiones en el manejo nutricional del $\mathrm{N}, \mathrm{P}$ y $\mathrm{K}$.

\section{LITERATURA CITADA}

Balasu-bramanian V, Morales AC, Cruz RT, Thiyagarajan TM, Nagarajan R, Babu M, et al. (2000) Adaptation of the chlorophyll meter (SPAD) technology for real-time $\mathrm{N}$ management in rice: a review. International Rice Research Institute 5: 25-26.

Bojovic B, Stojanovic J (2005) Chlorophyll and carotenoid content in wheat cultivars as a function of mineral nutrition. Archives of Biological Sciences 57: 283-290.

Calatayud A, Barreno E (2004) Response to ozone in two lettuce varieties on chlorophyll a fluorescence, photosynthetic pigments and lipid peroxidation. Plant Physiology and Biochemistry 42: 549-555.

Daughtry CST, Walthall CI, Kim MS, Brown de Colstoun E, McMurtrey JE (2000) Estimating corn leaf chlorophyll concentration from leaf and canopy reflectance. Remote Sensing of Environment 74: 229239.

Demotes-Mainard S, Boumaza R, Meyer S, Cerovic ZG (2008) Indicators of nitrogen status for ornamental 
woody plants based on optical measurements of leaf epidermal polyphenol and chlorophyll contents. Scientia Horticulturae 115: 377-385.

Grossman A, Takahashi H (2001) Macronutrient utilization by photosynthetic eukaryotes and the fabric of interactions. Annual Review of Plant Physiology and Plant Molecular Biology 52: 163-210.

Hawkesford M, Horst W, Kichey T, Lambers H, Schjoerring J, Skrumsager I, White P (1995) Functions of macronutrients. In: Marschner H (Ed.) Mineral nutrition of higher plants. Third Edition. Academic Press Inc., San Diego CA. pp 135-190.

Kraiser T, Gras DE, Gutiérrez AG, González B, Gutiérrez RA (2011) A holistic view of nitrogen acquisition in plants. Journal of Experimental Botany 62: 1455-1466.

Kumar Tewari R, Kumar P, Sharma N (2007) Oxidative stress and antioxidant responses in Young leaves of mulberry plants grown under nitrogen, phosphorus or potassium deficiency. Journal of Integrative Plant Biology 49: 313-322.

Lamrani Z, Belakbir A, Ruiz JM, Ragala L, López-Cantarero I, Romero L (1996) Influence of nitrogen, phosphorus, and potassium on pigment concentration in cucumber leaves. Communications in Soil Science and Plant Analysis 27: 1001-1012.

Latsague M, Sáez P, Mora M (2014) Efecto de la fertilización con nitrógeno, fósforo y potasio, sobre el contenido foliar de carbohidratos, proteínas y pigmentos fotosintéticos en plantas de Berberidopsis corallina Hookf. Gayana Botánica 71: 37-42.

Ling N, Chen D, Guo H, Wei J, Bai Y, Shen Q, Hu S (2017) Differential responses of soil bacterial communities to long-term $\mathrm{N}$ and $\mathrm{P}$ inputs in a semi-arid steppe. Geoderma 292: 25-33.

Masoudi-Sadaghiani F, Babak AM, Zardoshti MR, Hassan RSM, Tavakoli A (2011) Response of proline, soluble sugars, photosynthetic pigments and antioxidant enzymes in potato (Solanum tuberosum L.) to different irrigation regimes in greenhouse condition. Australian Journal of Crop Science 5: 55-60.

Muñoz-Huerta RF, Guevara-Gonzalez RG, Contreras-Medina LM, Torres-Pacheco I, Prado-Olivarez J, Ocampo-Velazquez RV (2013) A review of methods for sensing the nitrogen status in plants: advantages, disadvantages and recent advances. Sensors 13: 10823-10843.

Naeem M, Khan MMA (2009) Phosphorus ameliorates crop productivity, photosynthesis, nitrate reductase activity and nutrient accumulation in coffee senna (Senna occidentalis L.) under phosphorus-deficient soil. Journal of Plant Interactions 4: 145-153.

Parry MAJ, Flexas J, Medrano H (2005) Prospects for crop production under drought: research priorities and future directions. Annals Applied Biology 147: 211-226.

Raghothama KG, Karthikeyan AS (2005) Phosphate acquisition. Plant and Soil 274: 37-49.

Raghothama KG (1999) Phosphate acquisition. Annual Review of Plant Physiology and Plant Molecular Biology 50: 665-693.

Rubio G, Lynch JP (2007) Compensation among root classes of Phaseolus vulgaris L. Plant and Soil 290: 307-321.

Salinas R, Sánchez E, Ruíz JM, Lao MT, Romero L (2012) Producción de biomasa y rendimiento en judía verde (Phaseolus vulgaris L.) cv. Strike en respuesta a la fertilización fosforada. Phyton, International Journal of Experimental Botany 81: 35-39. 
Salinas R, Sánchez E, Ruíz JM, Lao MT, Romero L (2013) Phosphorus levels influence plasma membrane $\mathrm{H}+$-ATPase activity and $\mathrm{K}^{+}, \mathrm{Ca}^{2+}$, and $\mathrm{Mg}^{2+}$ assimilation in green bean. Communications in Soil Science and Plant Analysis 44: 456-464.

Sadzawka RA, Carrasco MA, Demanet R, Flores P, Grez Z, Mora G, et al. (2007) Métodos de análisis de tejidos vegetales. Instituto de Investigaciones Agropecuarias. Serie Actas INIA No. 40. Chile. http://www2.inia.cl/medios/biblioteca/serieactas/NR34664.pdf. Fecha de consulta: 10 de abril de 2018.

Sánchez E, Soto JM, García PC, López-Lefebre LR, Rivero RM, Ruiz JM, Romero L (2000) Phenolic compounds and oxidative metabolism in Green bean plants under nitrogen toxixity. Australian Journal of Plant Physiology 27: 973-978.

Sánchez E, Rivero RM, Ruiz JM, Romero L (2004) Changes in biomass, enzymatic activity and protein concentration in roots and leaves of green bean plants (Phaseolus vulgaris L. Cv. Strike) under high $\mathrm{NH}_{4} \mathrm{NO}_{3}$ application rates. Scientia Horticulturae 99: 237-248.

Sánchez E, Ávila-Quezada G, Gardea AA, Muñoz E, Ruiz JM, Romero L (2009) Nitrogen metabolism in roots and leaves of green bean plants exposed to different phosphorus doses. Phyton, International Journal of Experimental Botany 78: 11-16.

Tanaka Y, Sasaki N, Ohmiya A (2008) Biosynthesis of plant pigments: anthocyanins, betalains and carotenoids. The Plant Journal 54: 733-749.

Tejada-Zarco PJ, Miller JR, Morales A, Berjon A, Aguera J (2004) Hyperspectral indices and simulation models for chlorophyll estimation in open-canopy tree crops. Remote Sensing of Environment 90: 463-476.

Torres-Netto A, Campostrini E, Goncalves de Oliveira J, Bressan-Smith RE (2005) Photosynthetic pigments, nitrogen, chlorophyll a flurorescence and SPAD-502 readings in coffee leaves. Scientia Horticulturae 104: 199-209.

Vance CP, Uhde-Stone C, Allan DL (2003) Phosphorus acquisition and use: critical adaptations by plants for securing a nonrenewable resource. New Phytologist 157: 423-447.

Wellburn AR (1994) The special determination of chlorophylls $a$ and $b$ as well as total carotenoids using various solvents with spectrophotometers of different resolution. Journal of Plant Physiology 144: 307-313.

Xu HX, Weng XY, Yang Y (2007) Effect of phosphorus deficiency on the photosynthetic characteristics of rice plants. Russian Journal of Plant Physiology 54: 741-748.

Yaryura P, Cordon G, León M, Kerber N, Pucheu N, Rubio G, García A, Lagorio MG (2009) Effect of phosphorus deficiency on reflectance and chlorophyll fluorescence of cotyledons of oilseed rape (Brassica napus L.). Journal of Agronomy and Crop Science 195: 186-196.

Zhang X, Huang G, Bian X, Zhao Q (2013) Effects of root interaction and nitrogen fertilization on the chlorophyll content, root activity, photosynthetic characteristics of intercropped soybean and microbial quantity in the rhizosphere. Plant, Soil and Environment 59: 80-88. 